\title{
X-RAY GENERATION DURING PIEZOELECTRIC LIGHTER OPERATION IN VACUUM
}

\author{
O. O. Ivashchuk, ${ }^{1,3,6}$ A. V. Shchagin, ${ }^{1,2}$ A. S. Kubankin, ${ }^{1,3}$ A. A. Klenin, ${ }^{1}$ A. N. Oleinik,, 4 \\ Yu. V. Grigor'ev, ${ }^{5}$ A. S. Kumskov, ${ }^{5}$ and E. V. Bolotov ${ }^{1}$
}

Translated from Steklo i Keramika, No. 12, pp. 34 - 38, December, 2020.

\begin{abstract}
The results of experimental studies on the generation of $\mathrm{x}$-rays when operating a piezoelectric kitchen lighter in a vacuum are presented. For the first time, a new method for increasing the intensity of x-ray radiation in the piezoelectric effect in a high vacuum through the use of an additional electron emitter is proposed and demonstrated. The maximum energy of x-ray bremsstrahlung reaches $14 \mathrm{keV}$. This means that electrons are accelerated in vacuum in the field of a piezoelectric ceramic to energy of at least $14 \mathrm{keV}$.
\end{abstract}

Key words: piezoelectric lighter, generation of $\mathrm{x}$-rays, electron emitter, piezoelectric ceramic.

Recently, several new approaches have been proposed to the development of miniature electron accelerators and x-ray generators with energy up to tens of $\mathrm{keV}$ without the use of external high-voltage power supplies.

One such compact source is an electron accelerator and an x-ray generator based on: the pyroelectric effect in vacuum, using single crystals (as a rule, lithium niobate or lithium tantalate) $[1-3]$ or ceramics $[4,5]$ and the triboelectric effect in vacuum [6,7]. Pyroelectric sources can be used: as an emitter of electrons and ions $[8,9]$ and a neutron generator [10-13]; in installations for measuring x-ray fluorescence [14]; in mass spectrometry [15]; in an electron probe microanalyzer [16]; and, in a cathode-luminescence spectrometer [17]. Research is being conducted on the operation of a pyroelectric source in a pulsed mode [18, 19]. Recently, the generation of $\mathrm{x}$-rays with cutoff energy $12 \mathrm{keV}$ was discovered when a piezoelectric ceramic transformer operated in vacuum [20 - 22]. The possibility of using dielectric channels to control beams of accelerated electrons with energy up to $10 \mathrm{keV}$ is demonstrated in [23]. An electron accelerator

1 International Research Laboratory of Radiation Physics, Belgorod National Research University, Belgorod, Russia.

2 National Scientific Center Kharkov Institute of Physics and Technology, Kharkov, Ukraine.

3 P. N. Lebedev Physical Institute of the Russian Academy of Sciences, Moscow, Russia.

4 Royal Holloway, University of London, Egham Hill, UK.

5 A. V. Shubnikov Institute of Crystallography, FSRC Crystallography and Photonics, Russian Academy of Sciences, Moscow, Russia. based on the piezoelectric effect in vacuum is of scientific and practical interest. Such an accelerator was first proposed in [24]. The generation of $x$-rays with limiting energy up to $60 \mathrm{keV}$ in such an accelerator was observed on changing of the mechanical load applied to samples of the piezoelectric ceramic lead zirconate-titanate-borate [24] and quartz single crystals [25] in vacuum.

In the present work we investigate the generation of $\mathrm{x}$-rays when the piezoelectric lighter mechanism [26] operates in a vacuum, which provides sufficient mechanical pressure on piezoelectric samples in vacuum to generate $\mathrm{x}$-rays without resorting to a bulky deformation machine system $[24,25]$. This embodiment of the piezoelectric generator can be considered as a miniature device in which the conversion of mechanical energy into $\mathrm{x}$-ray energy occurs. It is assumed that the enhancement of the $\mathrm{x}$-ray generation effect during operation of a piezoelectric lighter in a high vacuum of about $10^{-6}$ Torr $\left(1.33 \times 10^{-4} \mathrm{~Pa}\right)$ can be observed when using an additional source of electrons, which can be a filament [18].

\section{EXPERIMENT}

A schematic diagram of the experimental setup is shown in Fig. 1. Experimental studies were performed in the radiation physics laboratory at Belgorod State National Research University. A mechanically driven piezoelectric lighter with an additional high-voltage electrode, a filament, and an x-ray spectrometer detector are arranged in the vacuum chamber. 


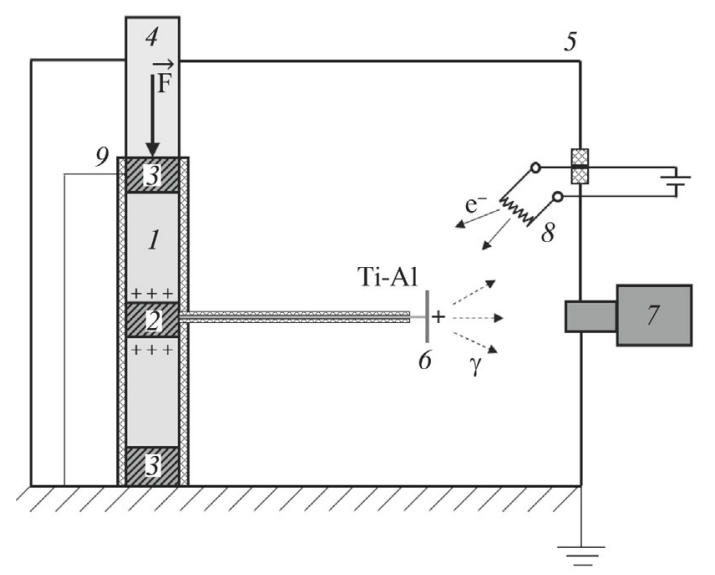

Fig. 1. Schematic diagram of the experimental setup: 1) an assembly of two piezoelectric ceramic samples made of the material TsTBS-3M; 2 ) central high-voltage electrode; 3 ) grounded metal disks; 4 ) metal cylinder providing a force $F ; 5$ ) vacuum chamber; 6 ) additional high-voltage electrode, titanium-aluminum plate; 7 ) semiconductor x-ray detector; 8 ) electron emitter; 9) dielectric casing.

A piezoelectric lighter [26] (Monolit plant, Vitebsk, Belarus) with a common body, high-voltage wire, and metal tube, which are missing in its design, was installed in a vacuum chamber (Fig. 2). Its main structural elements are two cylindrical piezoelectric samples made of lead zirconate-titanate-borate ceramic (TsTBS-3M), $6.4 \mathrm{~mm}$ in diameter and $15 \mathrm{~mm}$ high, installed coaxially and mechanically in series between the power lever and the plate of the drive mechanism of the piezoelectric lighter.

The power lever is capable of providing a mechanical pressure of about several tens of megapascals on piezoelectric samples when a force of the order of several tens of newtons is applied to it from a mechanical manipulator on the outside of the vacuum chamber. A central high-voltage electrode made in the form of a metal disk $6.4 \mathrm{~mm}$ in diameter and $1 \mathrm{~mm}$ high was situated between the piezoelectric cylinders. The back surfaces of each piezoelectric were fixed on metal grounded disks connected to both parts of the drive mechanism (power arm and metal plate), with a diameter and height equal to those of the central high voltage electrode.

For additional insulation in order to avoid surface breakdowns, the assembly of the piezoelectric samples and the central electrode in the construction of the piezoelectric lighter is situated in a dielectric casing. The spontaneous polarization vectors of each piezoelectric are collinear but oppositely directed. During normal operation of the lighter in air a breakdown in the discharge chamber occurs at a voltage of several kilovolts and no dangerous x-ray radiation occurs.

The filament, which served as an electron emitter in the experiment, was located at a distance of about $200 \mathrm{~mm}$ from the central high-voltage electrode. We used a $3.5 \mathrm{~V}$ flashlight filament. The bulb glass was removed.

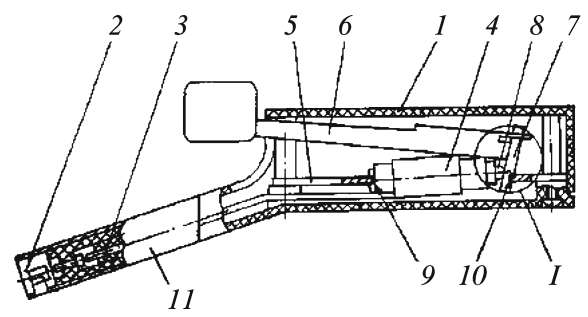

Fig. 2. Scheme of the longitudinal section of a piezoelectric lighter from [26]: 1 ) common body; 2 ) discharge chamber; 3 ) high-voltage wire; 4 ) block of piezoelectric elements; 5 ) plane-parallel plate of the drive mechanism; 6) manipulator lever; 7) power lever; 8 ) metal ball; 9, 10 ) metal disks; 11 ) metal tube.

An additional high-voltage titanium-aluminum alloy electrode was placed near the filament at a distance of about $10 \mathrm{~mm}$, which was connected to the central high-voltage electrode of the lighter with an insulated conductor. The distance between the electrode and the detector was about $20 \mathrm{~mm}$.

An x-ray spectrometer was used to register x-rays. It consisted of an Amptek SDD-100 semiconductor silicon detector with a $100 \mu \mathrm{m}$ thick beryllium input window and a PX5 digital pulse processor. The spectrometer was calibrated with the help of an Amptek COOL-X pyroelectric X-ray source using tantalum L lines [27]. The peak time of the spectrometer was $1 \mu \mathrm{sec}$, and its collimated area was $17 \mathrm{~mm}^{2}$.

In experimental studies the spontaneous polarization vector of each piezoelectric was directed from the central high-voltage electrode to the grounded metal disks.

\section{RESULTS}

The experiments were performed at chamber pressure $3 \times 10^{-6}$ Torr $\left(4 \times 10^{-4} \mathrm{~Pa}\right)$. A mechanical pressure of about several tens of megapascals, controlled by a manipulator from the outside of the vacuum chamber, was applied to an assembly of two piezoelectric samples situated in vacuum. The bottom of the vacuum chamber served as the base of the drive mechanism of the piezoelectric lighter.

The compression time was about $10 \mathrm{sec}$. Thus, a positive potential due to the piezoelectric effect was formed on the titanium-aluminum plate. An electric field accelerated the free electrons from the residual gas toward the titanium-aluminum plate. X-rays were generated when electrons were decelerated on the atoms of the plate. The x-ray spectrum measured during the compression of an assembly of two piezoelectric samples in vacuum and for the next $20 \mathrm{sec}$ is displayed in Fig. $3 a$. Note that the cutoff energy of the x-ray radiation is $9 \mathrm{keV}$, and the total number of detected quanta within $30 \mathrm{sec}$ (total measurement time) is 3752 .

It is worth noting that the intensity of x-ray radiation recorded at the first stage of the experiment is rather low, about 125 quanta recorded per second in the entire spectrum. This 

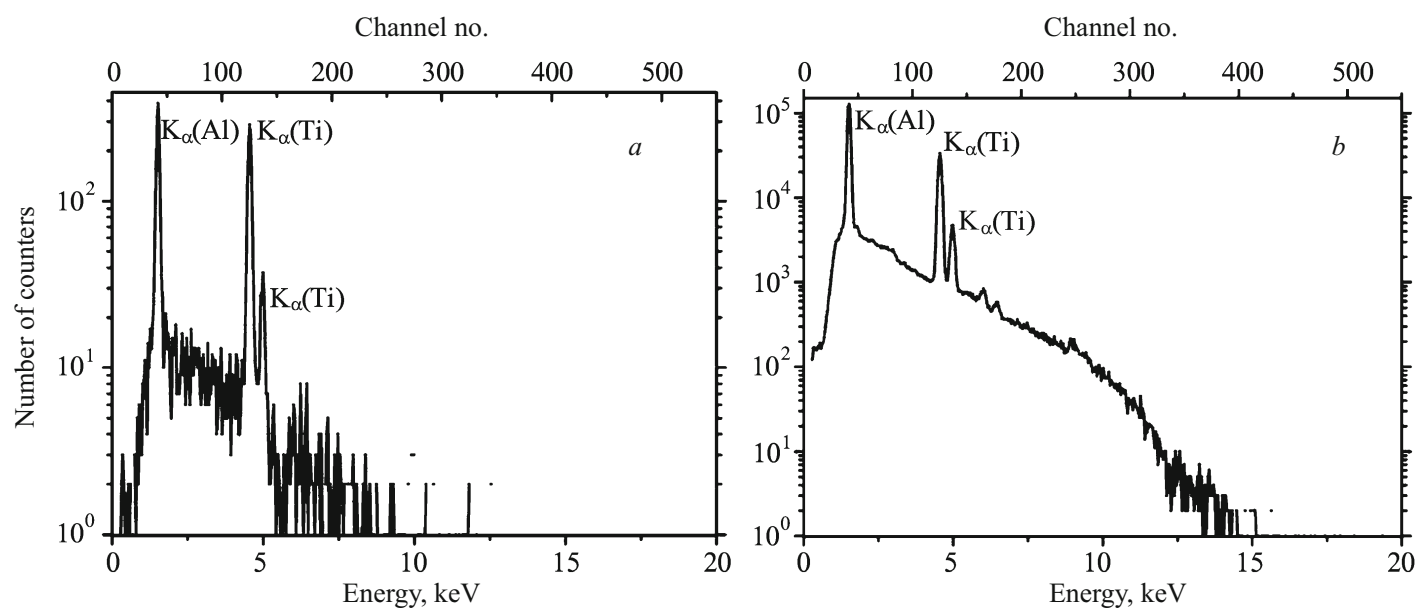

Fig. 3. X-ray spectra measured on compression of the piezoelectric ceramic TsTBS-3M with the help of the pie-zoelectric lighter mechanism with the filament turned off $(a)$ and with compression and subsequent switching on of the filament $(b)$ at a residual gas pressure of about $10^{-6}$ Torr $\left(1.3 \times 10^{-4} \mathrm{~Pa}\right)$.

low intensity can be explained by the low concentration of free charge carriers.

We used an additional emitter of electrons in order to increase the intensity.

At the completion of the first stage of the experiment a voltage of about $3.5 \mathrm{~V}$ was immediately applied to the filament. The filament emitted electrons, which gradually discharged the titanium-aluminum plate over the next $60 \mathrm{sec}$, which was accompanied by intense generation of $\mathrm{x}$-rays.

The x-ray spectrum measured during $10 \mathrm{sec}$ of compression of the piezoelectric ceramic, the next $20 \mathrm{sec}$, and with the filament turned on for $60 \mathrm{sec}$ is shown in Fig. $3 b$. The spectrum clearly shows the characteristic lines of x-ray radiation with energies of $1.5,4.5$ and $4.9 \mathrm{keV}$, corresponding to the $\mathrm{K}_{\alpha}$ and $\mathrm{K}_{\beta}$ atoms of aluminum and titanium (included in the plate), against the background of smoothly decaying bremsstrahlung radiation. The total number of detected quanta during $90 \mathrm{sec}$ is 878,140 . The cutoff energy of $\mathrm{x}$-ray radiation is $14 \mathrm{keV}$. This means that the energy of the electrons accelerated to the titanium-aluminum plate is at least $14 \mathrm{keV}$, and the maximum positive potential at the high-voltage electrode is at least $14 \mathrm{kV}$. When the filament is turned on, the $\mathrm{x}$-ray intensity rises sharply and reaches 14,573 quanta per second. The time for complete discharge of the crystal in this case is about $30 \mathrm{sec}$.

\section{CONCLUSIONS}

In summary, the possibility of $\mathrm{x}$-ray generation when operating a piezoelectric lighter in vacuum has been experimentally confirmed. For the first time, an additional electron emitter was used in a piezoelectric accelerator. The additional electron emitter makes it possible to increase the intensity by two orders of magnitude and the maximum energy of $\mathrm{x}$-ray radiation by approximately 1.6 times. It is worth noting that the proposed electron accelerator based on the piezo- electric effect in vacuum is capable of generating intense $\mathrm{x}$-ray radiation within a few seconds. The results of the performed experimental studies confirm the possibility of creating a miniature piezoelectric electron accelerator, based on the piezoelectric effect, up to an energy of at least $14 \mathrm{keV}$ and an x-ray generator with a limiting energy of about $14 \mathrm{keV}$ with an increased radiation intensity. In such devices mechanical energy is converted through the piezoelectric effect into the energy of accelerated electrons and the energy of $\mathrm{x}$-ray radiation.

This work was performed with financial support from the competitive part of the state task on the creation and development of laboratories, project No. FZWG-2020-0032 (2019-1569) and using equipment from the Center for Collective Use at the Federal Research Center for Crystallography and Photonics with the support of the Ministry of Education and Science of the Russian Federation (project RFMEFI62119X0035).

\section{REFERENCES}

1. J. D. Brownridge, "Pyroelectric x-ray generator," Nature, 358, $287-288$ (1992).

2. J. D. Brownridge and S. Raboy, "Investigations of pyroelectric generation of x-rays," J. Appl. Phys., 86, 640 (1997).

3. J. Geuther and Y. Danon, "High-energy x-ray production with pyroelectric crystals," J. Appl. Phys., 97, 104916 (2005).

4. K. A. Vokhmyanina, O. O. Ivashchuk, V. Yu. Ionidi, et al., "Possibility of using the piezoceramic PZT-19 in pyroelectric x-ray generators," Steklo Keram., No. 11, 27 - 31 (2016); K. A. Vokhmyanina, O. O. Ivashchuk, V. Yu. Ionidi, et al., "Possibility of using the piezoceramic PZT-19 in pyroelectric x-ray generators," Glass Ceram., 73(11 - 12), 415 - 419 (2016).

5. A. V. Shchagin, V. S. Miroshnik, V. I. Volkov, and A. N. Oleinik, "Ferroelectric ceramics in a pyroelectric accelerator," Appl. Phys. Lett. 107, 233505 (2015). 
6. J. R. Hird, C. G. Camara, and S. J. Putterman, "A triboelectric X-ray source," Appl. Phys. Lett. 68, 133501 (2011).

7. C. G. Camara, J. V. Escobar, J. R. Hird, and S. J. Putterman, "Correlation between nanosecond X-ray flashes and stick-slip friction in peeling tape," Nature, 455, 1089 - 1092 (2008).

8. M. Hockley and Z. Huang, "Strong electron and ion emissions induced by a pyroelectric crystal," J. Appl. Phys., 113, 034902 (2013).

9. V. I. Nagaichenko, V. S. Miroshnik, A. M. Yegorov and A. V. Shchagin, "Research of the spectra of the charged particles beam in the pyroelectric accelerator," Vopr. Atom. Nauki Tekh., Ser. Yaderno-Fiz. Issled., No. 2(53), $34-39$ (2010); V. I. Nagaichenko, V. S. Miroshnik, A. M. Yegorov and A. V. Shchagin, "Research of the spectra of the charged particles beam in the pyroelectric accelerator," Probl. Atomic Sci. Technol., Ser. Nucl. Phys. Invest., No. 2(53), 34 - 39 (2010).

10. B. Naranjo, J. K. Gimzewski, and S. Putterman, "Observation of nuclear fusion driven by a pyroelectric crystal," Nature, $\mathbf{4 3 4}$, 1115 - 1117 (2005).

11. J. Geuther, Ya. Danon, and F. Saglime, "Nuclear reactions induced by a pyroelectric accelerator," Phys. Rev. Lett., 96, 054803 (2006).

12. W. Tornow, S. M. Shafroth, and J. D. Brownridge, "Evidence for neutron production in deuterium gas with a pyroelectric crystal without tip," J. Appl. Phys., 104, 034905 (2008).

13. D. Gillich, A. Kovanen, and B. Herman, "Pyroelectric crystal neutron production in a portable prototype vacuum system," Nucl. Instrum. Methods Phys. Res. Sect. A, 602, 306 (2009).

14. J. Kawai, et al., "X-ray fluorescence analysis with a pyroelectric X-ray generator," X-Ray Spectrom., 34, 521 (2005).

15. E. L. Neidholdt and J. L. Beauchamp, "Compact ambient pressure pyroelectric ion source for mass spectrometry," Anal. Chem., 79, 3945 (2007).

16. S. Imashuku and K. Wagatsuma, "Portable pyroelectric electron probe microanalyzer with a spot size of $40 \mu \mathrm{m}$," Rev. Sci. Instrum., 88, 023117 (2017).
17. S. Imashuku, N. Fuyuno, J. Kawai, and K. Hanasaki, "Portable rare-earth element analyzer using pyroelectric crystal," Rev. Sci. Instrum., 84, 126105 (2013).

18. O. O. Ivashchuk, A. V. Shchagin, A. S. Kubankin, et al., "Pyroelectric accelerator and x-ray source in pulsed mode," JINST, 15, Ñ02002 (2020).

19. L. Orlikov, K. M. Mambetowa, S. I. Arestov, and S. M. Shandarov, "Pulse source of electrons based on the pyroeffect," in: 7th International Congress on Energy Fluxes and Radiation Effects (EFRE) [in Russian], Tomsk (2020), pp. 359 - 363.

20. B. Gall, S. D. Kovaleski, J. A. VanGordon, et al., "Investigation of the piezoelectric effect as a means to generate x-rays," IEEE Trans. Plasma Sci., 41, 106 - 111 (2013).

21. O. O. Ivashchuk, A. V. Shchagin, V. S. Miroshnik, et al., "Ceramic piezoelectric transformer in vacuum for acceleration of electrons and production of x-rays," Materials, 11, 1188 (2018).

22. A. V. Shchagin, V. S. Miroshnik, V. I. Volkov, et al., "Piezoelectric transformer aided x-ray generation in vacuum," Steklo Keram., No. 11, 24 - 26 (2017); A. V. Shchagin, V. S. Miroshnik, V. I. Volkov, et al., "Piezoelectric transformer aided x-ray generation in vacuum," Glass Ceram., 74(11 - 12), $404-405$ (2017).

23. K. A. Vokhmyanina, V. S. Sotnikova, A. A. Kaplii, et al., "Possibility of using dielectric channels as deflecting systems for controlling accelerated electron beams," Steklo Keram., No. 10, 15 - 18 (2017); K. A. Vokhmyanina, V. S. Sotnikova, A. A. Kaplii, et al., "Possibility of using dielectric channels as deflecting systems for controlling accelerated electron beams," Glass Ceram., 74(9 - 10), 355 - 357 (2017).

24. O. O. Ivashchuk, A. V. Shchagin, A. S. Kubankin, et al., "Piezoelectric Accelerator," Sci. Rep., 8, 816488 (2018).

25. O. O. Ivashchuk, A. V. Shchagin, A. S. Kubankin, et al., "Quartz accelerator of charged particles," Probl. Atomic Sci. Technol., Ser. Nucl. Phys. Invest., 3(127), 59 (2020).

26. A. V. Kukuruza and G. A. Deryabin, Piezoelectric Lighter, Pat. BY $935 U$ [in Russian] (2003).

27. Pyroelectric X-Ray Source COOL-X, Amptek; URL: http://www.amptek.com/pdf/coolx.pdf. 